\title{
Measurement of Thromboxane Biosynthesis in Health and Disease
}

\author{
Carlo Patrono* and Bianca Rocca \\ Department of Pharmacology, Catholic University School of Medicine, Rome, Italy
}

Thromboxane (TX) $A_{2}$ is a chemically unstable lipid mediator involved in several pathophysiologic processes, including primary hemostasis, atherothrombosis, inflammation, and cancer. In human platelets, $\mathrm{TXA}_{2}$ is the major arachidonic acid derivative via the cyclooxygenase (COX)-1 pathway. Assessment of platelet TXA ${ }_{2}$ biosynthesis can be performed ex vivo through measurement of serum $\mathrm{TXB}_{2}$, an index of platelet COX-1 activity, as well as in vivo through measurement of urinary enzymatic metabolites, a non-invasive index of platelet activation. This article reviews the main findings of four decades of clinical investigation based on these analytical approaches, focusing on the measurement of $\mathrm{TXA}_{2}$ metabolites to characterize the pathophysiologic role of transiently or persistently enhanced platelet activation and to describe the clinical pharmacology of COX-1 inhibition in health and disease.

\section{OPEN ACCESS}

Edited by:

G. Enrico Rovati,

University of Milan, Italy

Reviewed by:

Anthony Ashton,

University of Sydney, Australia

Jiiang-Huei Jeng,

National Taiwan University,

Taiwan

*Correspondence:

Carlo Patrono

carlo.patrono@unicatt.it

Specialty section: This article was submitted to Inflammation Pharmacology,

a section of the journal

Frontiers in Pharmacology

Received: 03 June 2019 Accepted: 27 September 2019

Published: 30 October 2019

Citation:

Patrono C and Rocca B (2019) Measurement of Thromboxane Biosynthesis in Health and Disease.

Front. Pharmacol. 10:1244.

doi: 10.3389/fphar.2019.01244
Keywords: thromboxane, prostanoids biosynthesis, aspirin, cardiovasular disease, platelet activation

\section{INTRODUCTION}

The pictorial description in 1962 of the aggregation of blood platelets by ADP, using a novel device called the Born aggregometer (Born, 1962), paved the way to quantitative assessment of platelet inhibition in vitro and ex vivo (reviewed by Born and Patrono, 2006). However, the possibility of establishing a mechanistic link between the inhibition of platelet prostanoid formation by aspirin (Smith and Willis, 1971) and inhibition of platelet aggregation had to wait the discovery of a novel pro-aggregating and vasoconstrictor prostanoid, thromboxane $(\mathrm{TX}) \mathrm{A}_{2}$, as the major arachidonic acid derivative in human platelets (Hamberg et al., 1975). This discovery allowed the development of appropriate analytical tools to investigate platelet $\mathrm{TXA}_{2}$ biosynthesis and its inhibition by aspirin in human health and disease (reviewed by Born and Patrono, 2006). TXA 2 is a pro-thrombotic, chemically unstable prostanoid, mostly synthesized via cyclooxygenase (COX)-1 and released by activated platelets (reviewed by Davì and Patrono, 2007). Two different biomarkers were characterized independently to assess $\mathrm{TXA}_{2}$ biosynthesis ex vivo, i.e., whole-blood $\mathrm{TXB}_{2}$ production (Patrono et al., 1980), and in vivo, i.e., urinary $\mathrm{TXB}_{2}$ metabolite excretion (Roberts et al., 1981) (Figure 1). By raising a specific antibody against $\mathrm{TXB}_{2}$, the chemically stable and biologically inactive hydrolysis product of $\mathrm{TXA}_{2}$, we were able to measure the time-dependent synthesis and release of platelet $\mathrm{TXA}_{2}$ induced by endogenous thrombin generated during whole-blood clotting and to demonstrate its suppression by low doses of aspirin (Patrono et al., 1980). The pioneering work of John Oates and his associates at Vanderbilt University was responsible for the development of a non-invasive approach to investigating prostanoid biosynthesis in man, based on the measurements of urinary prostanoid metabolites. In 1981, they reported the conversion of systemically infused $\mathrm{TXB}_{2}$ into 20 enzymatic derivatives, which were identified in the urine of a single healthy volunteer by gas chromatography/mass spectrometry (GC/MS) (Roberts et al., 1981). The availability of these analytical tools paved the way for investigating $\mathrm{TXA}_{2}$ biosynthesis in health and disease and 


\section{Ex Vivo}

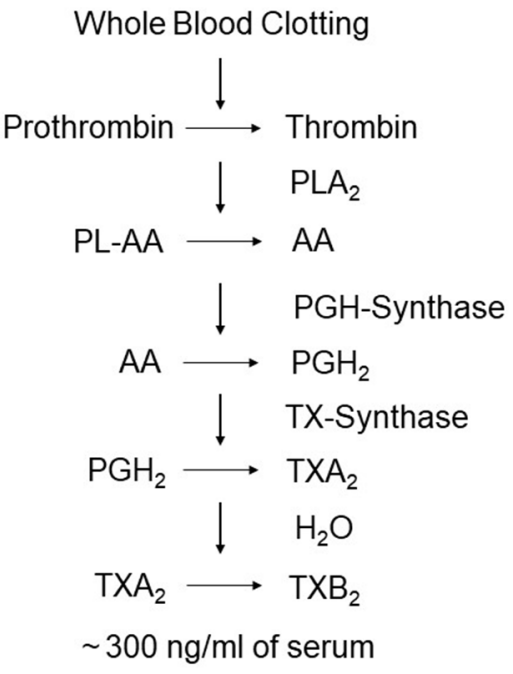

In Vivo

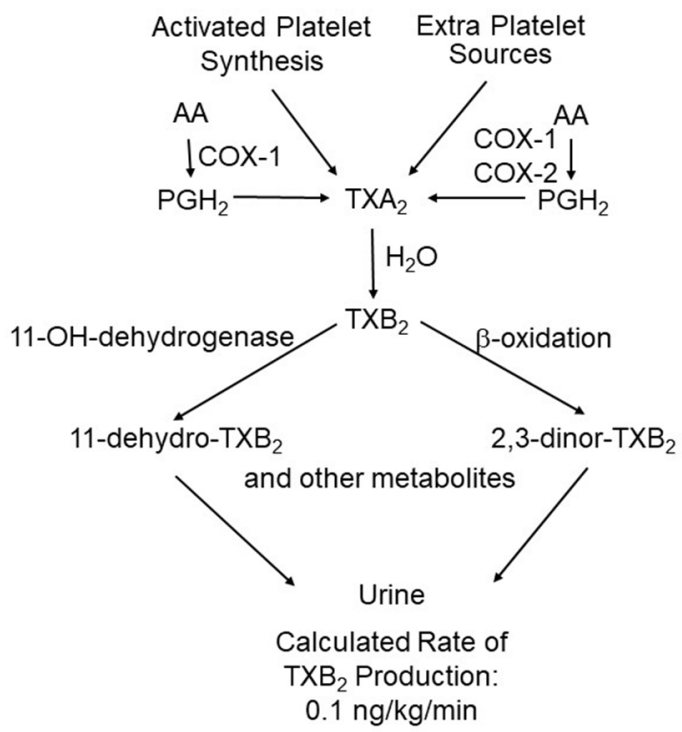

FIGURE 1 | Assessment of platelet thromboxane (TX) $\mathrm{A}_{2}$ biosynthesis ex vivo and in vivo. The left panel depicts the chain of enzymatic reactions triggered by

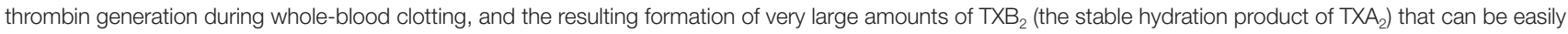
measured in serum as a sensitive and specific index of platelet COX-1 activity. The right panel depicts the metabolic fate of TXA in vivo and the calculated rate of its production in healthy subjects on the basis of $\mathrm{TXB}_{2}$ infusions and measurement of its major urinary metabolites, 11-dehydro-TXB $\mathrm{B}_{2}$ and 2,3-dinor-TXB $\mathrm{B}_{2}$. The latter represent a non-invasive index of platelet activation in vivo. Modified and redrawn from Patrono et al. (2013).

its selective, cumulative inhibition by low-dose aspirin, that eventually led to its development as an antiplatelet drug for the treatment and prevention of atherothrombosis (reviewed by Patrono, 1994).

This article reviews the main findings of four decades of clinical investigation based on these analytical approaches, focusing on the measurement of $\mathrm{TXA}_{2}$ metabolites in vivo and ex vivo as indexes of platelet activation and COX-1 activity, respectively, with emphasis on the authors' contribution to the resulting pathophysiological and pharmacological developments.

\section{URINARY THROMBOXANE METABOLITE EXCRETION AS A NON-INVASIVE BIOMARKER OF PLATELET ACTIVATION IN VIVO}

In 1981, Roberts et al. reported the GC/MS characterization of 20 enzymatic metabolites of systemically infused $\left[{ }^{3} \mathrm{H}_{8}\right]$ $\mathrm{TXB}_{2}$. Two major series of metabolites were identified based on a ring structure. One series retained the original $\mathrm{TXB}_{2}$ hemiacetal ring and included two metabolites, 2,3-dinor- $\mathrm{TXB}_{2}$ (the most abundant urinary metabolite) and 2,3,4,5-tetranor$\mathrm{TXB}_{2}$, which were products of beta-oxidation (Roberts et al., 1981). The second group of derivatives was formed as a result of dehydrogenation of the hemiacetal alcohol group at C-11, and included 16 metabolites (Roberts et al., 1981). Among the compounds resulting from this single transformation, 11-dehydro- $\mathrm{TXB}_{2}$ was the most abundant urinary metabolite (Roberts et al., 1981). In their seminal paper in the Journal of
Biological Chemistry, Roberts et al. accurately predicted the potential value of this analytical approach: "Since thromboxanes are released in substantial quantities from aggregating platelets, quantification of in vivo thromboxane production may provide a means to assess in vivo platelet aggregation and lead to a better understanding of the role of platelets in the pathophysiology of many cardiovascular diseases. It may also provide a means to assess the in vivo efficacy of anti-platelet drug therapy" (Roberts et al., 1981). Important limitations of this study were represented by a single high rate of $\mathrm{TXB}_{2}$ infusion and a single healthy subject being infused, precluding assessment of the linearity of conversion of $\mathrm{TXB}_{2}$ into its major enzymatic derivatives, as well as of the interindividual variability in the prevalence of the two main pathways of its metabolic transformation.

Together with Garret FitzGerald and Ian Blair, we reexamined the metabolic fate of $\mathrm{TXB}_{2}$ entering the systemic circulation, by measuring the urinary excretion of 2,3-dinor- $\mathrm{TXB}_{2}$ during the infusion of exogenous $\mathrm{TXB}_{2}$, in four aspirin-pretreated healthy volunteers randomized to receive 6-h i.v. infusions of vehicle alone and $\mathrm{TXB}_{2}$ at $0.1,1.0$, and $5.0 \mathrm{ng} \cdot \mathrm{kg}^{-1} \cdot \mathrm{min}^{-1}$ (Patrono et al., 1986). Plasma $\mathrm{TXB}_{2}$ and urinary 2,3-dinor-TXB ${ }_{2}$ were measured before, during, and up to $24 \mathrm{~h}$ after the infusions and in aspirin-free periods. Aspirin treatment suppressed baseline urinary 2,3-dinor- $\mathrm{TXB}_{2}$ excretion by $80 \%$, consistent with a predominant platelet source of the parent compound. The fractional excretion of 2,3-dinor- $\mathrm{TXB}_{2}$ was independent of the rate of $\mathrm{TXB}_{2}$ infusion, over a 50-fold dose range, and averaged 5.3\% $\pm 0.8 \%$ (Patrono et al., 1986). Insertion of 2,3-dinor- $\mathrm{TXB}_{2}$ excretion rates measured in aspirin-free periods into the linear relationship between the doses of infused $\mathrm{TXB}_{2}$ and the amounts of metabolite excreted in excess of control values 
permitted estimation of the rate of entry of endogenous $\mathrm{TXB}_{2}$ into the circulation as $0.11 \mathrm{ng} \cdot \mathrm{kg}^{-1} \cdot \mathrm{min}^{-1}$ (Patrono et al., 1986). Upon discontinuing $\mathrm{TXB}_{2}$ infusion, its rate of disappearance from the systemic circulation was linear over the first $10 \mathrm{~min}$ with an apparent half-life of $7 \mathrm{~min}$. This resulted in a maximal estimate of the plasma concentration of endogenous $\mathrm{TXB}_{2}$ of $2.0 \mathrm{pg} / \mathrm{ml}$, i.e., much lower than had been previously reported (Patrono et al., 1986). This finding argued for a local nature of $\mathrm{TXA}_{2}$ synthesis and action, as previously suggested for prostacyclin ( $\mathrm{PGI}_{2}$ ) (FitzGerald et al., 1981). Similar to the endothelial synthesis of $\mathrm{PGI}_{2}$, the maximal $\mathrm{TXA}_{2}$ biosynthetic capacity of human platelets greatly exceeds its actual production in vivo. Thus, the platelets of $1 \mathrm{ml}$ of whole blood clotted for $1 \mathrm{~h}$ in vitro can synthesize and release a similar amount of $\mathrm{TXB}_{2}$ as that secreted into the systemic circulation in vivo during the same time (Patrono et al., 1980; Patrono et al., 1986) (Figure 1), a finding that may help explain the unusual requirement for greater than $97 \%$ inhibition of $\mathrm{TXA}_{2}$ biosynthetic capacity to maximally inhibit $\mathrm{TXA}_{2}$-dependent platelet function (Reilly and FitzGerald, 1987; Santilli et al., 2009) (see below).

However, because of obvious safety concerns, it had not been possible to investigate the metabolic fate of TXA $\mathrm{T}_{2}$ in humans, and it remained to be determined whether the enzymatic transformation of $\mathrm{TXB}_{2}$ to its major urinary metabolites accurately reflected $\mathrm{TXA}_{2}$ metabolism in vivo. Thus, together with Joe Rokach and his colleagues at Merck Frosst Research Laboratories, Patrignani et al. (1989) compared the metabolic handling of exogenously infused $\mathrm{TXA}_{2}$ and $\mathrm{TXB}_{2}$ in the cynomolgus monkey. The main finding of this study was that $\mathrm{TXA}_{2}$ and $\mathrm{TXB}_{2}$ are metabolized to 2,3-dinor- $\mathrm{TXB}_{2}$ and 11-dehydro- $\mathrm{TXB}_{2}$ with similar fractional conversion rates, thereby suggesting that $\mathrm{TXA}_{2}$ is hydrolyzed non-enzymatically to $\mathrm{TXB}_{2}$ prior to enzymatic degradation via the beta-oxidation and 11-OH-dehydrogenase pathways, and that the resulting urinary metabolites provide a quantitative index of TXA 2 biosynthesis in vivo (Patrignani et al., 1989).

Because previous estimates of the rate of entry of $\mathrm{TXB}_{2}$ into the human systemic circulation had been based on monitoring the beta-oxidation pathway of $\mathrm{TXB}_{2}$ metabolism (Patrono et al., 1986), Ciabattoni et al. (1989) went on to measure the urinary excretion of immunoreactive 11-dehydro- $\mathrm{TXB}_{2}$ and 2,3-dinor$\mathrm{TXB}_{2}$ (Ciabattoni et al., 1987) during the infusion of exogenous $\mathrm{TXB}_{2}$ over a 50 -fold dose range in healthy volunteers, with the same protocol of the previous study (Patrono et al., 1986). The fractional elimination of both metabolites was independent of the rate of $\mathrm{TXB}_{2}$ infusion and averaged $6.0 \%$ to $7.0 \%$, demonstrating that urinary 11-dehydro- $\mathrm{TXB}_{2}$ is at least as abundant a conversion product of exogenously infused $\mathrm{TXB}_{2}$ as 2,3-dinor- $\mathrm{TXB}_{2}$ (Ciabattoni et al., 1989) Furthermore, the study of Ciabattoni et al. (1987) showed that this analytical approach could detect changes in the urinary excretion of immunoreactive 11-dehydro$\mathrm{TXB}_{2}$ associated with simulated short-term increases of $\mathrm{TXB}_{2}$ release into the human circulation (Ciabattoni et al., 1987).

Transient increases in the excretion of 2,3-dinor- $\mathrm{TXB}_{2}$ and 11-dehydro- $\mathrm{TXB}_{2}$ were described in patients with acute coronary syndromes and interpreted as reflecting repeated episodes of platelet activation (Fitzgerald et al., 1986; Vejar et al., 1990). Transient changes in $\mathrm{TXA}_{2}$ biosynthesis detected in patients with unstable angina were accompanied by concomitant increases in $\mathrm{PGI}_{2}$ biosynthesis, as reflected by urinary 2,3-dinor-6-keto$\mathrm{PGF}_{1 \alpha}$ excretion, suggesting a counter-regulatory endothelial activation in this setting (Fitzgerald et al., 1986). In contrast, patients with chronic stable angina did not display increased $\mathrm{TXA}_{2}$ biosynthesis, both under resting conditions and following exercise-induced myocardial ischemia (Fitzgerald et al., 1986). Biochemical evidence of episodic platelet activation in the setting of acute coronary syndromes was consistent with the postmortem findings of Michael Davies and Erling Falk that suggested dynamic thrombotic events occurring over a disrupted plaque in the coronary vessels of patients dying after a diagnosis of unstable angina (reviewed by Falk et al., 1995; Davì and Patrono, 2007). These results provided a rationale for testing the efficacy and safety of low-dose aspirin in acute coronary syndromes, a clinical setting in which antiplatelet therapy reduced the risk of major atherothrombotic complications by approximately $50 \%$ (reviewed by Patrono et al., 2005). Episodic increases in $\mathrm{TXB}_{2}$ metabolite excretion were also characterized in patients with acute ischemic stroke (Koudstaal et al., 1993; van Kooten et al., 1997), though with a lower frequency and shorter duration than in acute coronary syndromes (Fitzgerald et al., 1986; Vejar et al., 1990), perhaps reflecting the heterogeneity of mechanisms responsible for ischemic stroke (Albers et al., 2004). Low-dose aspirin (50 $\mathrm{mg}$ daily) largely suppressed 11-dehydro- $\mathrm{TXB}_{2}$ excretion in this setting, reflecting the predominant platelet origin of $\mathrm{TXA}_{2}$ biosynthesis (Koudstaal et al., 1993). Enhanced platelet activation was independently associated with stroke severity on admission (van Kooten et al., 1997). Patients with a transient ischemic attack were characterized by infrequent episodes of platelet activation, suggesting that enhanced $\mathrm{TXA}_{2}$ biosynthesis was not secondary to cerebral ischemia (Koudstaal et al., 1993).

Persistent platelet activation, as reflected by persistently enhanced urinary excretion of $\mathrm{TXB}_{2}$ metabolites, was reported by different Groups in patients with a variety of cardiovascular risk factors that accelerate atherogenesis, including cigarette smoking (Nowak et al., 1987), type-2 diabetes mellitus (Davì et al., 1990), hypercholesterolemia (Davì et al., 1992), homozygous homocystinuria (Di Minno et al., 1993), and hypertension (Minuz et al., 2002). Because enhanced urinary excretion of 11-dehydro$\mathrm{TXB}_{2}$ in diabetes mellitus might reflect either an abnormality in the biosynthesis of $\mathrm{TXA}_{2}$ or a shift in its metabolic fate through the two main enzymatic pathways of degradation, Davi et al. (1990) investigated the fractional conversion of infused $\mathrm{TXB}_{2}$ to urinary 11-dehydro- $\mathrm{TXB}_{2}$ in subjects with type-2 diabetes mellitus. Their finding of a linear conversion of infused $\mathrm{TXB}_{2}$ to urinary 11-dehydro- $\mathrm{TXB}_{2}$ over a 50 -fold range of infusion rates, with a fractional elimination similar to that previously described in healthy subjects (Ciabattoni et al., 1989), was consistent with enhanced excretion of 11-dehydro- $\mathrm{TXB}_{2}$ reflecting a change in the biosynthesis of $\mathrm{TXA}_{2}$ rather than an alteration in its enzymatic transformations (Davì et al., 1990).

Urinary prostanoid metabolites, such as 11-dehydro- $\mathrm{TXB}_{2}$, do not reflect a specific site of prostanoid biosynthesis. To characterize the potential platelet versus non-platelet sources of TXA $_{2}$ biosynthesis, Davi et al. (1990) used the unique property of aspirin to produce selective, cumulative acetylation of platelet cyclooxygenase (COX)-1 when it is given in low doses once daily 
(Patrignani et al., 1982). Renal COX-isozymes, which have been involved in enhanced $\mathrm{TXA}_{2}$ production in patients with systemic lupus erythematosus (Patrono et al., 1985), are not inhibited by low-dose aspirin to any detectable extent (Patrignani et al., 1982; Pierucci et al., 1989). The profound reduction in urinary 11-dehydro- $\mathrm{TXB}_{2}$ excretion that was found in subjects with type- 2 diabetes mellitus after they received $50 \mathrm{mg}$ of aspirin daily for 1 week and the platelet turnover-dependent return to a pre-treatment excretion rate over the next 10 days following aspirin discontinuation were consistent with a prevailing role for platelets as the source of TXA 2 biosynthesis in this setting (Davi et al., 1990).

Persistently enhanced urinary excretion of $\mathrm{TXB}_{2}$ metabolites has been reported in the vast majority of patients with myeloproliferative neoplasms, such as essential thrombocythemia (ET) and polycythemia vera (PV) (reviewed by Patrono et al., 2013). As shown in Figure 2, the urinary excretion rates of 11-dehydro-TXB ${ }_{2}$ measured in untreated ET (Rocca et al., 1995) and PV patients (Landolfi et al., 1992) are comparable to the rate measured in patients with unstable angina (Fitzgerald et al., 1986; Vejar et al., 1990) and higher than values of metabolite excretion associated with a variety cardiovascular risk factors (Nowak et al., 1987; Davì et al., 1990; Davì et al., 1992; Di Minno et al., 1993; Minuz et al., 2002). The finding of persistently enhanced TXA 2 biosynthesis in PV patients and its suppression by low-dose aspirin (Landolfi et al., 1992) provided a rationale for testing the efficacy and safety of this antiplatelet strategy in PV (Landolfi et al., 2004). Based on the positive results of the ECLAP
(European Collaboration on Low-dose Aspirin in Polycythemia vera) trial (Landolfi et al., 2004), primary prophylaxis with lowdose aspirin (81-100 mg daily) is currently recommended for PV patients (Tefferi et al., 2018).

Urinary 11-dehydro- $\mathrm{TXB}_{2}$ excretion has been investigated as a potential biomarker of the future risk of major vascular events in aspirin-treated, high-risk patients enrolled in the Heart Outcomes Prevention Evaluation (HOPE) trial, by using a nested case-control design (Eikelboom et al., 2002). After adjustment for baseline differences, the odds for the composite end-point of myocardial infarction, stroke, or cardiovascular death increased with each increasing quartile of baseline urinary 11-dehydro- $\mathrm{TXB}_{2}$, with patients in the upper quartile having approximately 2 -fold higher risk than those in the lower quartile (Eikelboom et al., 2002). This finding was largely confirmed by a similar substudy of the Clopidogrel for High Atherothrombotic Risk and Ischemic Stabilization, Management and Avoidance (CHARISMA) trial (Eikelboom et al., 2008). However, because both HOPE and CHARISMA were mostly secondary prevention trials, an important limitation of these analyses is represented by the lack of a control group of subjects not treated with aspirin. Furthermore, the extent of biological variation in urinary 11-dehydro- $\mathrm{TXB}_{2}$ excretion rate, as well as the intrasubject variability in its reduction by low-dose aspirin are currently unknown, but could potentially limit the value of this biomarker to predict the risk of future cardiovascular events in an individual aspirin-treated patient. We are currently investigating the potential predictive value of urinary 11-dehydro- $\mathrm{TXB}_{2}$ excretion

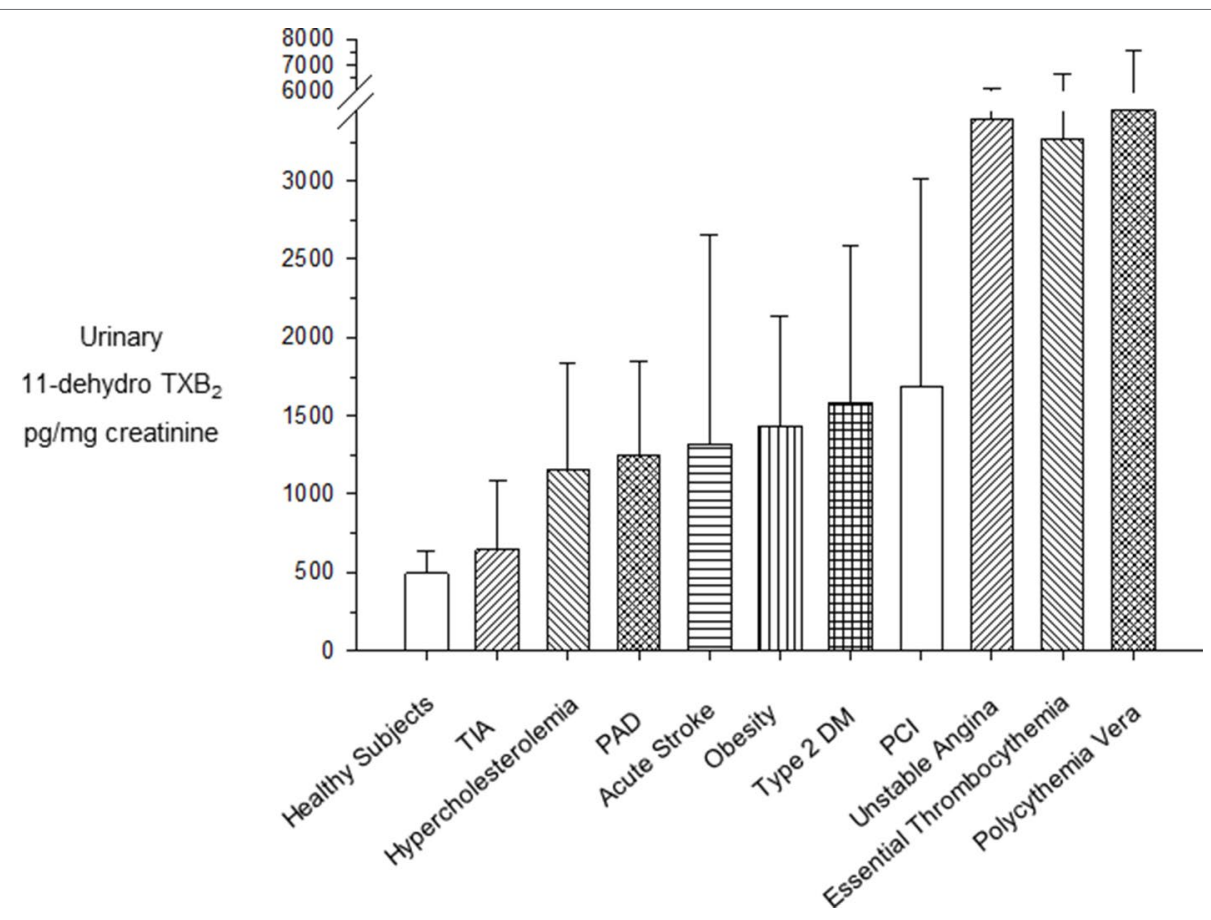

FIGURE 2 | Urinary excretion rates of 11-dehydro-TXB in clinical settings at high cardiovascular risk. Mean ( \pm standard deviation) or median (interquartile range) urinary excretion rates of 11-dehydro- $\mathrm{TXB}_{2}$ in clinical settings characterized by high cardiovascular risk. PAD, peripheral arterial disease; PCI, percutaneous coronary intervention; T2DM, type 2 diabetes mellitus; TIA, transient ischemic attack. Modified and redrawn from Patrono et al. (2013). 
in a large sample of A Study of Cardiovascular Events in Diabetes (ASCEND) that randomized 15,480 adults with diabetes mellitus to long-term treatment with low-dose aspirin or placebo (ASCEND Study Collaborative Group, 2018).

Recently, the platelet origin of urinary thromboxane metabolite (TXM) excretion was challenged by the case report of a single patient with end-stage renal failure requiring dialysis who carried a rare genetic mutation in $\mathrm{CPLA}_{2 \alpha}$ (cytosolic phospholipase $\mathrm{A}_{2}$ ), resulting in dramatically reduced urinary TXM and $\mathrm{PGI}_{2}$ metabolite (PGIM) excretion rates, with recovery of "normal" urinary levels of these prostanoid metabolites after kidney transplantation (Mitchell et al., 2018). The authors' conclusion was that "urinary PGIM and TXM can be derived exclusively by the kidney without contribution from $\mathrm{PGI}_{2}$ made by endothelial cells or $\mathrm{TXA}_{2}$ by platelets in the systemic circulation" (Mitchell et al., 2018). However, the authors did not investigate the metabolic disposition of $\mathrm{TXB}_{2}$ and $\mathrm{PGI}_{2}$ in this patient, and no comparison was performed with renal failure and transplanted patients without the $\mathrm{CPLA}_{2 \alpha}$ genetic defect (Grosser et al., 2018). Moreover, it should be emphasized that in patients with systemic lupus erythematosus, who displayed enhanced renal synthesis of $\mathrm{TXA}_{2}$, higher urinary $\mathrm{TXB}_{2}$ excretion was associated with unchanged urinary excretion of 2,3-dinor- $\mathrm{TXB}_{2}$ (Patrono et al., 1985), suggesting that $\mathrm{TXA}_{2}$ produced by the kidney is mostly excreted unchanged into the urine and does not undergo systemic metabolism to TXM (Remuzzi et al., 1992).

\section{SERUM TXB ${ }_{2}$ AS A VALIDATED INDEX OF PLATELET COX-1 ACTIVITY}

The effects of aspirin on the activity of platelet COX-1 have been investigated through measurements of serum $\mathrm{TXB}_{2}$ (Patrono et al., 1980; Patrignani et al., 1982) and urinary metabolites of $\mathrm{TXB}_{2}$ (FitzGerald et al., 1983). Serum $\mathrm{TXB}_{2}$ reflects the maximal biosynthetic capacity of blood platelets to generate $\mathrm{TXA}_{2}$ in response to endogenously formed thrombin during whole-blood clotting, and its measurement has been used extensively to assess the human pharmacology of platelet COX-1 inhibition in health and disease (reviewed by Patrono et al., 2008). Three important features of aspirin pharmacodynamics were characterized by measurements of serum $\mathrm{TXB}_{2}$ in healthy subjects (Patrignani et al., 1982): i) the cumulative nature of the inactivation of platelet COX-1 by repeated daily low doses (20-40 mg) of aspirin; ii) the saturability of this effect with single doses as low as $100 \mathrm{mg}$; and iii) the relative selectivity for platelet COX-1 inhibition at low doses. Our Research Group at the Catholic University School of Medicine in Rome described timedependent, cumulative reduction in serum $\mathrm{TXB}_{2}$ (a product of platelet COX-1 activity), without statistically significant changes in urinary 6-keto- $\mathrm{PGF}_{1 \alpha}$ excretion (mostly a product of renal COX-2 activity), a reverse paradigm of selective COX-isozyme inhibition by low-dose aspirin (30 mg daily) in man (Patrignani et al., 1982) (Figure 3). Measurements of serum $\mathrm{TXB}_{2}$ were also instrumental in characterizing the presystemic nature of platelet COX-1 acetylation by aspirin, an important feature of aspirin pharmacodynamics contributing to its biochemical selectivity (Pedersen and FitzGerald, 1984). Thus, serum $\mathrm{TXB}_{2}$ was reduced by about $40 \%$ five min after the oral administration of a $20-\mathrm{mg}$ dose, before aspirin could be detected in the systemic circulation (Pedersen and FitzGerald, 1984).

It is important to realize that the serum $\mathrm{TXB}_{2}$ assay requires almost immediate whole-blood incubation at $37^{\circ} \mathrm{C}$ as a condition for optimal thrombin generation, arachidonic acid release and its sequential conversion by platelet COX-1 and TX-synthase to form $\mathrm{PGH}_{2}$ and $\mathrm{TXA}_{2}$, respectively. As a consequence of thrombininduced platelet activation, the blood concentration of $\mathrm{TXB}_{2}$ increases over $60 \mathrm{~min}$ from 1 to $2 \mathrm{pg} / \mathrm{ml}$ (true circulating plasma level in vivo) to 300 to $400 \mathrm{ng} / \mathrm{ml}$ (maximally stimulated, COX-1dependent production ex vivo) (Patrono et al., 1980). A variably delayed access to $37^{\circ} \mathrm{C}$ incubation, as well as different analytical methods to quantitate serum $\mathrm{TXB}_{2}$, may contribute to variable results in a multicenter setting (Frelinger et al., 2009; Reny et al., 2012). Petrucci et al. (2016) investigated whether a variable delay in $37^{\circ} \mathrm{C}$ incubation and/or analytical discrepancies may affect the assessment of aspirin pharmacodynamics based on serum $\mathrm{TXB}_{2}$

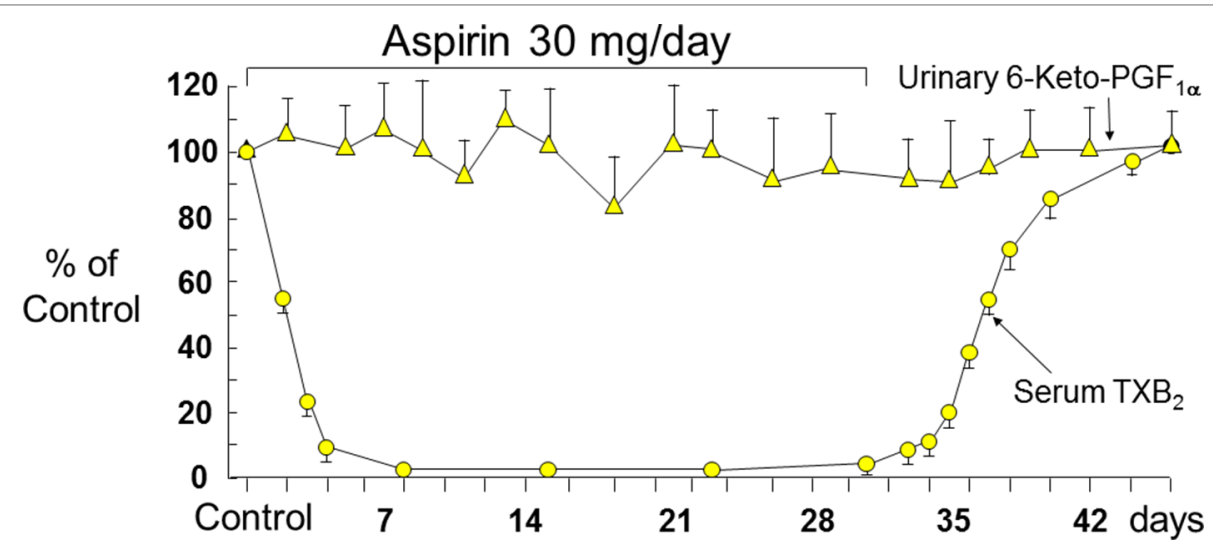

FIGURE 3 | Long-term effects of low-dose $\left(0.45 \mathrm{mg} / \mathrm{kg}\right.$ per day) aspirin on platelet $\mathrm{TXB}_{2}$ and renal $\mathrm{PGI}_{2}$ synthesis. Serum $\mathrm{TXB}{ }_{2}$ concentrations and urinary excretion of 6-keto-PGF $F_{1 a}$ were measured in three healthy subjects before, during, and after aspirin therapy. Mean values \pm SEM are plotted as percentage of control measurements performed prior to aspirin administration. The arrows indicate duration of daily aspirin therapy. Redrawn from Patrignani et al. (1982). 
determinations. They found that a longer than 5-min delay in the $37^{\circ} \mathrm{C}$ incubation of whole-blood samples may variably influence the assessment of platelet COX-1 inhibition by low-dose aspirin and confound the analysis of aspirin responsiveness in the clinical setting (Petrucci et al., 2016; De Stefano et al., 2018). In contrast, a GC/MS-validated immunoassay and liquid chromatographytandem mass-spectrometry yielded quite comparable $\mathrm{TXB}_{2}$ concentrations in the same serum samples (Petrucci et al., 2016).

The relationship between inhibition of platelet COX-1 activity, as reflected by serum $\mathrm{TXB}_{2}$, and arachidonic aciddependent platelet function assays (i.e., arachidonate-induced optical aggregation), and urinary 11-dehydro- $\mathrm{TXB}_{2}$ excretion is strikingly non-linear (Figure 4) (Reilly and FitzGerald, 1987; Santilli et al., 2009). Thus, platelet COX-1 activity must be nearly completely (>97\%) suppressed to fully inhibit in vivo platelet activation. Such stringent requirement may help explain the fact that the vast majority of traditional nonsteroidal antiinflammatory drugs (tNSAIDs), that are reversible inhibitors of platelet COX-1 with variable half-lives, are unable to achieve profound and persistent suppression of $\mathrm{TXA}_{2}$ biosynthesis, thereby unmasking their COX-2-dependent cardiovascular toxicity (reviewed by Patrono and Baigent, 2014). Although the variable COX-isozyme selectivity, half-life, daily dose, and duration of treatment of different COX-2 inhibitors could all influence the cardiovascular consequences of COX-2 inhibition, the dichotomous clinical read-outs of such inhibition are explained by the exponential relationship between inhibition of platelet COX-1 activity and suppression of $\mathrm{TXA}_{2}$-dependent platelet activation (Figure 4) (Patrono and Baigent, 2014). As depicted in Figure 4, NSAIDs inhibiting platelet COX-1 activity by $0 \%$ to $20 \%$ (e.g., highly selective COX-2 inhibitors, such as rofecoxib and etoricoxib), by $20 \%$ to $50 \%$ (e.g., COX-2 inhibitors with moderate COX-2 selectivity, such as diclofenac and celecoxib), or by $50 \%$ to $90 \%$ (most tNSAIDs, such as indomethacin and ibuprofen) will cause similarly modest suppression of $\mathrm{TXA}_{2}$-dependent platelet activation in vivo (Patrono and Baigent, 2014). Due to its longer half-life and modest COX-1 selectivity, naproxen $500 \mathrm{mg}$ twice daily may suppress platelet COX-1 activity by $>95 \%$ throughout the 12 -h dosing interval and reduce $\mathrm{TXA}_{2}$-dependent platelet activation in vivo to a similar extent as aspirin $100 \mathrm{mg}$ once daily (Capone et al., 2004; Capone et al., 2007).

Given the non-linear relationship depicted in Figure 4, platelet function assays, including urinary 11-dehydro- $\mathrm{TXB}_{2}$, do not accurately reflect the degree of platelet COX-1 inhibition (Pascale et al., 2012; Smith et al., 2012). Moreover, platelet function assays routinely used to measure aspirin response, are not necessarily related to its mechanism of action, display poor inter-assay agreement (Lordkipanidzé et al., 2007; Blais et al., 2009; Santilli et al., 2009; ), and give inconsistent results upon repeated measurements (Muir et al., 2009; Santilli et al., 2009). These methodological considerations may help explain the rise and fall of the aspirin "resistance" concept, typically defined as impaired platelet response to aspirin based on a single functional measurement performed at an often unspecified time point after

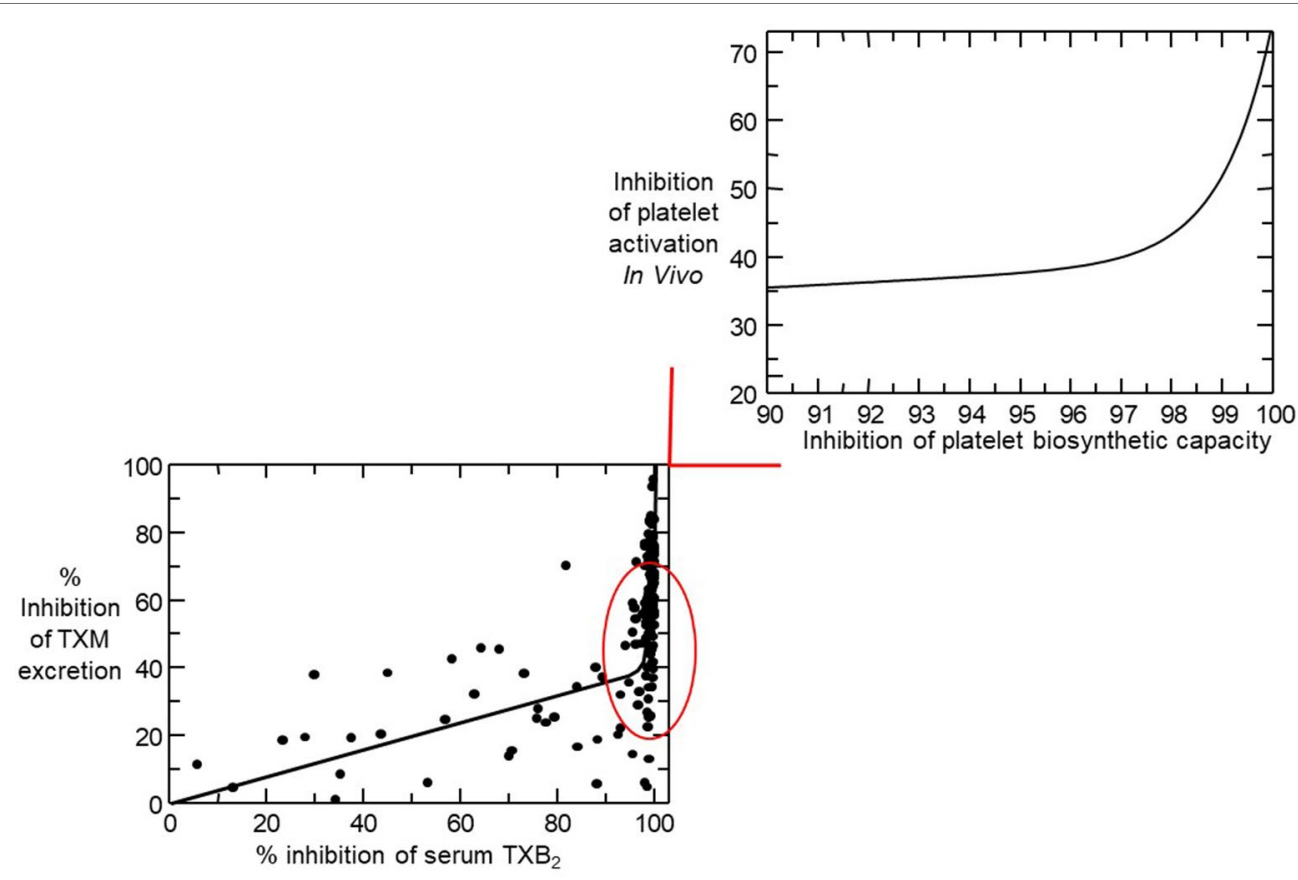

FIGURE 4 | Nonlinear relationship between inhibition of serum thromboxane (TX) $B_{2}$ and urinary 11-dehydro-TXB ${ }_{2}$ excretion. In the lower left panel, individual percentage inhibition values are depicted from all on-treatment and post-treatment measurements performed in 48 healthy subjects randomized to receive aspirin $100 \mathrm{mg}$ daily for 1 to 8 weeks. The nonlinear relationship between percent inhibition of serum $\mathrm{TXB}_{2}$ and urinary 11 -dehydro-TXB ${ }_{2}$ showed that for 0 to $97 \%$ of

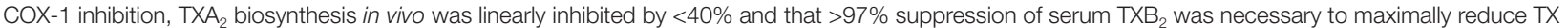
metabolite (TXM) excretion. The upper right panel represents a detail of the left panel and is based on mathematical modeling of the experimental data. Modified and redrawn from Santilli et al. (2009), with permission from the publisher. 
dosing, usually without a reliable assessment of compliance (Michelson et al., 2005; Rocca and Patrono, 2005). In a study of 48 healthy volunteers, repeated measurements of platelet aggregation in response to different agonists demonstrated that occasionally "resistant" subjects could be classified as "responders" when examined previously or subsequently (Santilli et al., 2009). Not surprisingly, the incidence of "resistance" ranged from $1 \%$ up to $65 \%$ in different studies, was assay-dependent, fluctuated over time, and remains of unproven clinical significance (reviewed by Patrono and Rocca, 2008).

In contrast to the uniform effectiveness of low-dose aspirin in suppressing platelet COX-1 activity in healthy individuals, some clinical conditions are associated with transient or persistent suboptimal platelet inhibition by a conventional once daily regimen of low-dose aspirin (Rocca and Patrono, 2005). These include patients following on-pump coronary artery bypass surgery (CABG) (Cavalca et al., 2017), patients with ET (Dragani et al., 2010; Dillinger et al., 2012), patients with coronary artery disease and the metabolic syndrome (Smith et al., 2012), and some patients with type-2 diabetes mellitus (Spectre et al., 2011; Rocca et al., 2012; Bethel et al., 2016). Under these circumstances, most patients display biochemical evidence of $\mathrm{TXA}_{2}$-dependent platelet activation in vivo (Davì et al., 1990; Rocca et al., 1995), a finding that may help explain impaired aspirin pharmacodynamics. More specifically, less-thanoptimal inactivation of platelet COX-1 could be a consequence of transiently (Cavalca et al., 2017) or persistently (Dragani et al., 2010) accelerated platelet renewal, or result from platelet activation-induced generation of hydroperoxides that may impair the acetylation of COX-1 by aspirin (Bala et al., 2008). The long-lasting duration of the antiplatelet effect of aspirin, despite its very short half-life, is explained by inactivation of COX-1 in bone-marrow platelet progenitors, as reflected by the 48 -h delay between aspirin withdrawal and initial recovery of unacetylated COX-1 (Burch et al., 1978) and TXA 2 biosynthetic capacity (Patrignani et al., 1982) in peripheral blood platelets. Thus, under conditions of normal thrombopoiesis, the efficacy of a short-lived drug given once daily reflects irreversible inactivation of a slowly renewable drug target (platelet COX-1) combined with an effect on bone-marrow platelet progenitors, leading to a new platelet progeny with largely non-functioning COX-1 for the vast majority of the 24-h dosing interval (Giaretta et al., 2017). However, reduced systemic bioavailability of aspirin, as may occur with some enteric-coated formulations (Maree et al., 2005) and in association with obesity (Petrucci et al., 2019), or faster renewal of platelet COX-1, as reported under conditions of altered megakaryopoiesis (Pascale et al., 2012), may shorten the duration of the antiplatelet effect of aspirin and dictate a more frequent dosing regimen (Patrono et al., 2013).

A standard once-daily regimen of low-dose aspirin administration cannot adequately suppress platelet $\mathrm{TXA}_{2}$ production, throughout the $24-\mathrm{h}$ dosing interval, in the vast majority of ET patients (Dragani et al., 2010; Dillinger et al., 2012; Pascale et al., 2012). An accelerated turnover of platelet COX-1, reflecting abnormal megakaryopoiesis, has been hypothesized in ET (Pascale et al., 2012; Rocca and Patrono, 2015; Giaretta et al., 2017). A higher daily fraction of newly released platelets with unacetylated COX-isozymes would account for incomplete inhibition, as well as timedependent recovery of $\mathrm{TXA}_{2}$-dependent platelet function during the standard 24-h dosing interval of low-dose aspirin administration (Dragani et al., 2010). Two relatively small studies in ET patients have shown that sub-optimal inhibition of platelet $\mathrm{TXA}_{2}$ production and $\mathrm{TXA}_{2}$-dependent platelet function can be largely rescued by a bid regimen of low-dose (100 mg) aspirin administration, but not by a higher dose (200$250 \mathrm{mg}$ ) given once daily (Dillinger et al., 2012; Pascale et al., 2012). The Aspirin Regimens in Essential Thrombocythemia Study (ARES) is a randomized, parallel-arm, dose-finding study recruiting $300 \mathrm{ET}$ patients to address two main questions (De Stefano et al., 2018). First, whether a bid or tid 100-mg aspirin regimen is more effective than the standard once daily regimen in inhibiting platelet $\mathrm{TXA}_{2}$ production, without a major impact on vascular $\mathrm{PGI}_{2}$ biosynthesis. Second, whether superior biochemical efficacy of a multiple versus single dosing low-dose aspirin regimen can be safely maintained over longterm follow-up (De Stefano et al., 2018).

Similarly, several independent studies have consistently shown the superior biochemical efficacy of a strategy based on shortening the dosing interval versus a strategy of maintaining or increasing the once daily dose of aspirin in patients with type-2 diabetes mellitus (Spectre et al., 2011; Rocca et al., 2012; Bethel et al., 2016), undergoing CABG (Paikin et al., 2015; Cavalca et al., 2017), or presenting with an acute coronary syndrome (Parker et al., 2019).

Obesity is associated with biochemical evidence of persistently enhanced platelet activation (Davì et al., 2002; Petrucci et al., 2019) and high risk of atherothrombotic complications (reviewed by Rocca et al., 2018). Recently, Rothwell et al. (2018) analyzed individual data of 117,279 subjects recruited into 10 primary prevention trials and reported that low doses of aspirin (75-100 $\mathrm{mg}$ ) were only effective in preventing major vascular events in subjects with body weight lower than $70 \mathrm{~kg}$, and had no benefit in the vast majority of men and nearly $50 \%$ of all women weighing 70 $\mathrm{kg}$ or more. In contrast, higher doses $(\geq 325 \mathrm{mg}$ ) of aspirin were only effective in subjects with body weight equal to or higher than $70 \mathrm{~kg}$ (Rothwell et al., 2018). Although the finding of effect modification by body weight has not been confirmed by some of the more recent aspirin trials (ASCEND Study Collaborative Group, 2018), these data appear consistent with the suggestion that the antiplatelet effect of aspirin, as reflected by serum $\mathrm{TXB}_{2}$ measurement, is influenced by body size (Maree et al., 2005). Increased body size, fat excess, and the associated changes in volume of distribution and liver function may all reduce the bioavailability of a lipophilic drug such as aspirin (Patrono and Rocca, 2017). Petrucci et al. (2019) recently reported measurements of serum $\mathrm{TXB}_{2}$ at the end of the 24-h dosing interval in 100 aspirin-treated subjects with a wide range of body mass index (BMI) and body weight values. A statistically significant exponential association was observed between body size, expressed as either BMI or body weight, and residual serum $\mathrm{TXB}_{2}$ values (Figure 5) (Petrucci et al., 2019). Thus, a standard, once-daily 100-mg aspirin regimen appears to be inadequate to fully inhibit platelet COX-1 activity throughout the 24-h dosing interval in moderately to severely obese subjects. However, based 
A

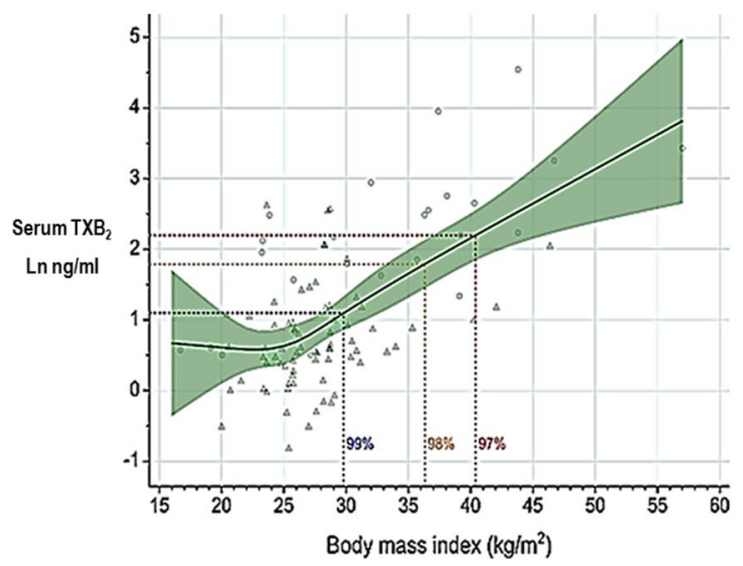

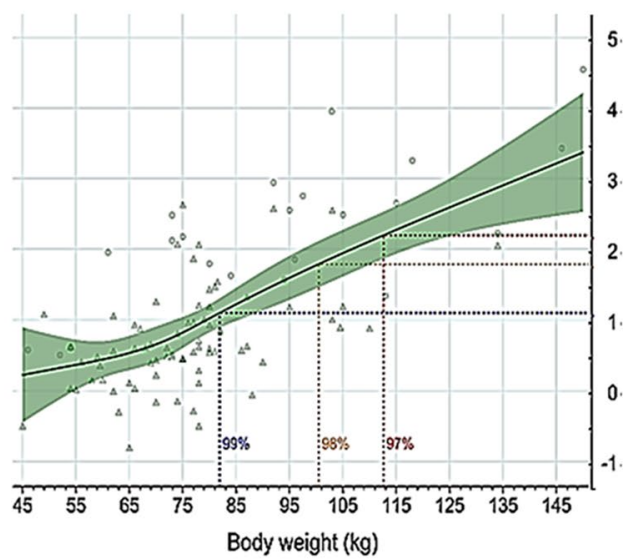

FIGURE 5 | Serum thromboxane (TX)B $B_{2}$ levels at $24 \mathrm{~h}$ after aspirin intake (100 mg once daily) vs body mass index or body weight. Log-transformed serum TXB levels measured $24 \mathrm{~h}$ after a witnessed aspirin intake are represented in relation to body mass index (panel A) and body weight (panel B). Triangles represent individual patients receiving aspirin for primary or secondary cardiovascular prevention, $n=71$; circles represent individual healthy subjects, $n=25$. Solid lines and green areas: predicted serum TXB ${ }_{2}$ levels and 95\% confidence intervals, respectively, as a function of increasing body mass index (panel $\mathbf{A}$ ) and body weight (panel

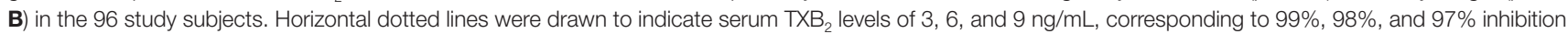
of platelet COX-1 activity, respectively, measured in healthy subjects. The vertical dotted lines were drawn to indicate on the abscissa scales the highest calculated values of body mass index (panel A) and body weight (panel B) compatible with these different levels of platelet COX-1 inhibition. Modified and redrawn from Petrucci et al., 2019, with permission from the publisher.

on the data depicted in Figure 5, one would not expect impaired aspirin pharmacodynamics in subjects with up to a body weight of $\sim 100 \mathrm{~kg}$ or a BMI of $35 \mathrm{~kg} / \mathrm{m}^{2}$, corresponding to obesity of class 2 or higher (Petrucci et al., 2019). In silico modeling of the antiplatelet pharmacodynamics of aspirin in this setting suggested that either doubling the once-daily dose or administering a lower dose (e.g., $85 \mathrm{mg}$ ) twice daily would be associated with adequate suppression of platelet TXA 2 production(Petrucci et al., 2019). The consensus opinion that "it is reasonable to double the daily dose or shorten the dosing interval (twice-daily) for BMI $\geq 40 \mathrm{~kg} / \mathrm{m}^{2}$ " expressed by the Working Group on Thrombosis of the European Society of Cardiology (Rocca et al., 2018) is consistent with these experimental findings and in silico modeling (Petrucci et al., 2019).

\section{CLINICAL IMPLICATIONS AND PERSPECTIVE}

If we look back at four decades of research on thromboxane biosynthesis and inhibition, the translational aspect of this research stands out in at least three areas. First, measurements of urinary TXM excretion, a non-invasive index of in vivo platelet activation, have been instrumental in identifying clinical conditions in which to assess the efficacy and safety of antiplatelet therapy. Notable examples are represented by acute coronary syndromes (Fitzgerald et al., 1986), acute ischemic stroke (Koudstaal et al., 1993), and polycythemia vera (Landolfi et al., 1992). The remarkable cardio-protective effects of lowdose aspirin in these settings (The RISC Group, 1990; Landolfi et al., 2004; Rothwell et al., 2016) are likely to reflect the important pathophysiologic role of transiently or persistently enhanced platelet activation unravelled by TXM measurements. Second, the development of serum $\mathrm{TXB}_{2}$ as a mechanismbased biomarker of platelet COX-1 inhibition (Patrono et al., 1980) has played a fundamental role in defining the human pharmacology of aspirin as an antiplatelet agent (Patrono, 1994). The results of a large series of randomized, placebo-controlled clinical trials of aspirin in the prevention of atherothrombosis in high-risk patients have confirmed the saturability of its antithrombotic effect at low doses (Antithrombotic Trialists' Collaboration, 2002), consistent with saturability of platelet COX-1 inactivation by low-dose aspirin (Patrignani et al., 1982; FitzGerald et al., 1983). Third, a comparison of the extent and duration of serum $\mathrm{TXB}_{2}$ reduction in response to different NSAIDs has allowed predicting a similar cardiovascular hazard of tNSAIDs and coxibs (Baigent and Patrono, 2003): "If the vascular consequences of endothelial COX-2 inhibition are modulated by profound and persistent blockade of platelet COX-1 activity, as indirectly implied by genetic and pharmacologic manipulations in mice (Cheng et al., 2002), then the cardiovascular effects of most traditional NSAIDs, which only incompletely and transiently inhibit platelet COX-1, may resemble those of selective COX-2 inhibitors." A prediction largely borne out by a tabular data meta-analysis published in 2006 (Kearney et al., 2006) and substantiated by an individual participant data meta-analysis of coxib and tNSAID trials in 2013 (CNT Collaboration et al., 2013).

Finally, as anticipated in the 1982 Journal of Clinical Investigation paper, "since the effect of low-dose aspirin is dependent upon platelet turnover as well as aspirin sensitivity 
of platelet and megakaryocyte cyclooxygenase, the adequacy of this therapeutic regimen might vary in different disease states" (Patrignani et al., 1982). More recent studies based on this methodological approach have largely confirmed this prediction both in specific clinical settings and by in silico modelling. Altogether, early and newer evidences have shown the desirability and practicality of adjusting the aspirin dosing interval based on two measurements of serum $\mathrm{TXB}_{2}$ at 12 and $24 \mathrm{~h}$ after dosing (Pascale et al., 2012; Rocca et al., 2012), a personalized approach that may be required under conditions

\section{REFERENCES}

Albers, G. W., Amarenco, P., Easton, J. D., Sacco, R. L., and Teal, P. (2004). Antithrombotic and thrombolytic therapy for ischemic stroke: the Seventh ACCP Conference on Antithrombotic and Thrombolytic Therapy. Chest 126 Suppl 3, 483S-512S. doi: 10.1378/chest.126.3_suppl.483S

Antithrombotic Trialists' Collaboration. (2002). Collaborative meta-analysis of randomised trials of antiplatelet therapy for prevention of death, myocardial infarction, and stroke in high risk patients. BMJ 324, 71-86. doi: 10.1136/ bmj.324.7329.71

ASCEND Study Collaborative Group, Bowman, L., Mafham, M., Wallendszus, K., Stevens, W., Buck, G., et al. (2018). Effects of Aspirin for Primary Prevention in Persons with Diabetes Mellitus. N. Engl. J. Med. 379, 1529-1539. doi: 10.1056/ NEJMoa 1804988

Baigent, C., and Patrono, C. (2003). Selective cyclooxygenase-2 inhibitors, aspirin and cardiovascular disease: a re-appraisal. Arthritis. Rheum. 48, 12-20. doi: 10.1002/art.10738

Bala, M., Chin, C. N., Logan, A. T., Amin, T., Marnett, L. J., Boutaud, O., et al. (2008). Acetylation of prostaglandin $\mathrm{H} 2$ synthases by aspirin is inhibited by redox cycling of the peroxidase. Biochem. Pharmacol. 75, 1472-1481. doi: 10.1016/j.bcp.2007.12.005

Bethel, M. A., Harrison, P., Sourij, H., Sun, Y., Tucker, L., Kennedy, I., et al. (2016). Randomized controlled trial comparing impact on platelet reactivity of twicedaily with once-daily aspirin in people with type 2 diabetes. Diabet. Med. 33, 224-230. doi: 10.1111/dme.12828

Blais, N., Pharand, C., Lordkipanidzé, M., Sia, Y. K., Merhi, Y., and Diodati, J. G. (2009). Response to aspirin in healthy individuals. Cross comparison of light transmission aggregometry, VerifyNow system, platelet count drop, thromboelastography (TEG) and urinary 11-dehydrothromboxane $\mathrm{B}(2)$. Thromb Haemost. 102, 404-411. doi: 10.1160/TH09-02-0126

Born, G. V. (1962). Aggregation of blood platelets by adenosine diphosphate and its reversal. Nature 194, 927-929. doi: 10.1038/194927b0

Born, G., and Patrono, C. (2006). Antiplatelet Drugs. Br. J. Pharmacol. 147, S241S251. doi: 10.1038/sj.bjp.0706401

Burch, J. W., Stanford, N., and Majerus, P. W. (1978). Inhibition of platelet prostaglandin synthetase by oral aspirin. J. Clin. Invest. 61, 314-319. doi: 10.1172/JCI108941

Capone, M. L., Tacconelli, S., Sciulli, M. G., Grana, M., Ricciotti, E., Minuz, P., et al. (2004). Clinical pharmacology of platelet, monocyte, and vascular cyclooxygenase inhibition by naproxen and low-dose aspirin in healthy subjects. Circulation 109, 1468-1471. doi: 10.1161/01.CIR.0000124715.27937.78

Capone, M. L., Tacconelli, S., Sciulli, M. G., Anzellotti, P., Di Francesco, L., Merciaro, G., et al. (2007). Human pharmacology of naproxen sodium. J. Pharmacol. Exp. Ther. 322, 453-460. doi: 10.1124/jpet.107.122283

Cavalca, V., Rocca, B., Veglia, F., Petrucci, G., Porro, B., Myasoedova, V., et al. (2017). On-pump cardiac surgery enhances platelet renewal and impairs aspirin pharmacodynamics: Effects of improved dosing regimens. Clin. Pharmacol. Ther. 102, 849-858. doi: 10.1002/cpt.702

Cheng, Y., Austin, S. C., Rocca, B., Koller, B. H., Coffman, T. M., Grosser, T., et al. (2002). Role of prostacyclin in the cardiovascular response to thromboxane A2. Science 296, 539-541. doi: 10.1126/science.1068711

Ciabattoni, G., Maclouf, J., Catella, F., FitzGerlad, G. A., and Patrono, C. (1987). Radioimmunoassay of 11-dehydro-thromboxane B2 in human plasma and urine. Biochim. Biophys. Acta 918, 293-397. doi: 10.1016/0005-2760(87)90233-5 of reduced systemic bioavailability of aspirin (Petrucci et al., 2019) or enhanced platelet turnover (Patrono et al., 2013).

\section{AUTHOR CONTRIBUTIONS}

$\mathrm{CP}$ has conceived and drafted the work. BR has contributed to the analysis and interpretation of the available literature in the field. $\mathrm{CP}$ and $\mathrm{BR}$ revised the paper critically for important intellectual content and provided approval for publication of the content.
Ciabattoni, G., Pugliese, F., Davì, G., Pierucci, A., Simonetti, B. M., and Patrono, C. (1989). Fractional conversion of thromboxane B2 to urinary 11-dehydro-thromboxane B2 in man. Biochim. Biophys. Acta 992, 66-70. doi: 10.1016/0304-4165(89)90051-2

CNT Collaboration, Bhala, N., Emberson, J., Merhi, A., Abramson, S., Arber, N., et al. (2013).Coxib and traditional NSAID Trialists'. Vascular and upper gastrointestinal effects of non-steroidal anti-inflammatory drugs: metaanalyses of individual participant data from randomised trials. Lancet 382, 769-779. doi: 10.1016/S0140-6736(13)60900-9

Davì, G., Catalano, I., Averna, M., Notarbartolo, A., Strano, A., Ciabattoni, G., et al. (1990). Thromboxane biosynthesis and platelet function in type II diabetes mellitus. N. Engl. J. Med. 322, 1769-1774. doi: 10.1056/NEJM199006213222503

Davì, G., Notarbartolo, A., Catalano, I., Averna, M., Barbagallo, C., Ciabattoni, G., et al. (1992). Increased thromboxane biosynthesis in type IIa hypercholesterolemia. Circulation 85, 1792-1798. doi: 10.1161/01. CIR.85.5.1792

Davì, G., Guagnano, M. T., Ciabattoni, G., Basili, S., Falco, A., Marinopiccoli, M., et al. (2002). Platelet activation in obese women: role of inflammation and oxidant stress. JAMA 288, 2008-2014. doi: 10.1001/jama.288.16.2008

Davì, G., and Patrono, C. (2007). Platelet activation and atherothrombosis. N. Engl. J. Med. 357, 2482-2494. doi: 10.1056/NEJMra071014

De Stefano, V., Rocca, B., Tosetto, A., Soldati, D., Petrucci, G., Beggiato, E., et al. (2018). The Aspirin Regimens in Essential Thrombocythemia (ARES) phase II randomized trial design - implementation of the serum thromboxane B2 assay as an evaluation tool of different dosing regimens in the clinical setting. Blood Cancer J. 8, 49. doi: 10.1038/s41408-018-0078-3

Dillinger, J. G., Sideris, G., Henry, P., Bal dit Sollier, C., Ronez, E., and Drouet, L. (2012). Twice daily aspirin to improve biological aspirin efficacy in patients with essential thrombocythemia. Thromb. Res. 129, 91-94. doi: 10.1016/j. thromres.2011.09.017

Dillinger, J. G., Drissa, A., Sideris, G., Bal dit Sollier, C., Voicu, S., Manzo Silberman, S., et al. (2012). Biological efficacy of twice daily aspirin in type 2 diabetic patients with coronary artery disease. Am. Heart J. 164, 600-606. doi: 10.1016/j.ahj.2012.06.008

Di Minno, G., Davì, G., Margaglione, M., Cirillo, F., Grandone, E., Ciabattoni, G., et al. (1993). Abnormally high thromboxane A2 biosynthesis in homozygous homocystinuria. Evidence for platelet involvement and probucol-sensitive mechanism. J. Clin. Invest. 92, 1400-1406. doi: 10.1172/JCI116715

Dragani, A., Pascale, S., Recchiuti, A., Mattoscio, D., Lattanzio, S., Petrucci, G., et al. (2010). The contribution of cyclooxygenase-1 and -2 to persistent thromboxane biosynthesis in aspirin-treated essential thrombocythemia: implications for antiplatelet therapy. Blood 115, 1054-1061. doi: 10.1182/ blood-2009-08-236679

Eikelboom, J. W., Hirsh, J., Weitz, J. I., Johnston, M., Yi, Q., and Yusuf, S. (2002). Aspirin-resistant thromboxane biosynthesis and the risk of myocardial infarction, stroke, or cardiovascular death in patients at high risk for cardiovascular events. Circulation 105, 1650-1655. doi: 10.1161/01. CIR.0000013777.21160.07

Eikelboom, J. W., Hankey, G. J., Thom, J., Bhatt, D. L., Steg, P. G., Montalescot, G., et al., et al. (2008). Incomplete inhibition of thromboxane biosynthesis by acetylsalicylic acid: determinants and effect on cardiovascular risk. Circulation 118, 1705-1712. doi: 10.1161/CIRCULATIONAHA.108.768283

Falk, E., Shah, P. K., Fuster, V., and Coronary plaque disruption. (1995). Circulation 92,657-671. doi: 10.1161/01.CIR.92.3.657 
FitzGerald, G. A., Brash, A. R., Falardeau, P., and Oates, J. A. (1981). Estimated rate of prostacyclin secretion into the circulation of normal man. J. Clin. Invest. 68 , 1272-1276. doi: 10.1172/JCI110373

FitzGerald, G. A., Oates, J. A., Hawiger, J., Maas, R. L., Roberts, L. J., 2nd, Lawson, J. A., et al. (1983). Endogenous biosynthesis of prostacyclin and thromboxane and platelet function during chronic administration of aspirin in man. J. Clin. Invest. 71, 676-688. doi: 10.1172/JCI110814

Fitzgerald, D. J., Roy, L., Catella, F., and FitzGerald, G. A. (1986). Platelet activation in unstable coronary disease. N. Engl. J. Med. 315, 983-989. doi: 10.1056/ NEJM198610163151602

Frelinger, A. L., 3rd, Li, Y., Linden, M. D., Barnard, M. R., Fox, M. L., Christie, D. J., et al. (2009). Association of cyclooxygenase-1-dependent and -independent platelet function assays with adverse clinical outcomes in aspirin-treated patients presenting for cardiac catheterization. Circulation 120, 2586-2596. doi: 10.1161/CIRCULATIONAHA.109.900589

Giaretta, A., Rocca, B., Di Camillo, B., Toffolo, G. M., and Patrono, C. (2017). In silico modeling of the antiplatelet pharmacodynamics of low-dose aspirin in health and disease. Clin. Pharmacol. Ther. 102, 823-831. doi: 10.1002/cpt.694

Grosser, T., Naji, A., and FitzGerald, G. A. (2018). Urinary prostaglandin metabolites: An incomplete reckoning and a flush to judgment. Circ. Res. 122, 537-539. doi: 10.1161/CIRCRESAHA.118.312616

Hamberg, M., Svensson, J., and Samuelsson, B. (1975). Thromboxanes: a new group of biologically active compounds derived from prostaglandin endoperoxides. Proc. Natl. Acad. Sci. U. S. A. 72, 2994-2998. doi: 10.1073/pnas.72.8.2994

Kearney, P. M., Baigent, C., Godwin, J., Halls, H., Emberson, J. R., and Patrono, C. (2006). Do selective cyclooxygenase-2 inhibitors and traditional non-steroidal anti-inflammatory drugs increase the risk of atherothrombosis? Meta-analysis of randomized trials. BMJ 332, 1302-1308. doi: 10.1136/bmj.332.7553.1302

Koudstaal, P. J., Ciabattoni, G., Van Gijn, J., Nieuwenhuis, H. K., De Groot, P., Sixma, J. J., et al. (1993). Increased thromboxane biosynthesis in patients with acute cerebral ischemia. Stroke 24, 219-223. doi: 10.1161/01.STR.24.2.219

Landolfi, R., Ciabattoni, G., Patrignani, P., Castellana, M. A. L., Pogliani, E., Bizzi, B., et al. (1992). Increased thromboxane biosynthesis in patients with polycythemia vera. Evidence of aspirin-suppressible platelet activation in vivo. Blood 80, 1965-1971.

Landolfi, R., Marchioli, R., Kutti, J., Gisslinger, H., Tognoni, G., Patrono, C., et al., et al. (2004). Efficacy and safety of low-dose aspirin in polycythemia vera. $N$. Engl. J. Med. 350, 114-124. doi: 10.1056/NEJMoa035572

Lordkipanidzé, M., Pharand, C., Schampaert, E., Turgeon, J., Palisaitis, D. A., and Diodati, J. G. (2007). A comparison of six major platelet function tests to determine the prevalence of aspirin resistance in patients with stable coronary artery disease. Eur. Heart J. 28, 1702-1708. doi: 10.1093/ eurheartj/ehm 226

Maree, A. O., Curtin, R. J., Dooley, M., Conroy, R. M., Crean, P., Cox, D., et al. (2005). Platelet response to low-dose enteric-coated aspirin in patients with stable cardiovascular disease. J. Am. Coll. Cardiol. 46, 1258-1263. doi: 10.1016/j.jacc.2005.06.058

Michelson, A. D, Cattaneo, M., Eikelboom, J. W., Gurbel, P., Kottke-Marchant, K., Kunicki, T. J., et al. (2005). Aspirin resistance: position paper of the Working Group on Aspirin Resistance. J. Thromb. Haemost. 3, 1309-1311.

Minuz, P., Patrignani, P., Gaino, S., Degan, M., Menapace, L., Tommasoli, R., et al. (2002). Increased oxidative stress and platelet activation in patients with hypertension and renovascular disease. Circulation 106, 2800-2805. doi: 10.1161/01.CIR.0000039528.49161.E9

Mitchell, J. A., Knowles, R. B., Kirkby, N. S., Reed, D. M., Edin, M. L., White, W. E., et al. (2018). Kidney transplantation in a patient lacking cytosolic phospholipase A2 proves renal origins of urinary PGI-M and TX-M. Circ. Res. 122, 555-559. doi: 10.1161/CIRCRESAHA.117.312144

Muir, A. R., McMullin, M. F., Patterson, C., and McKeown, P. P. (2009). Assessment of aspirin resistance varies on a temporal basis in patients with ischaemic heart disease. Heart 95, 1225-1229. doi: 10.1136/hrt.2008.150631

Nowak, J., Murray, J. J., Oates, J. A., and FitzGerald, G. A. (1987). Biochemical evidence of a chronic abnormality in platelet and vascular function in healthy individuals who smoke cigarettes. Circulation 76, 6-14. doi: 10.1161/01. CIR.76.1.6

Paikin, J. S., Hirsh, J., Ginsberg, J. S., Weitz, J. I., Chan, N. C., Whitlock, R. P., et al. (2015). Multiple daily doses of acetyl-salicylic acid (ASA) overcome reduced platelet response to once-daily ASA after coronary artery bypass graft surgery: a pilot randomised controlled trial. J. Thromb. Haemost. 13, 448-456. doi: $10.1111 /$ jth. 12832

Parker, W. A. E., Orme, R. C., Hanson, J., Stokes, H. M., Bridge, C. M., Shaw, P. A., et al. (2019). Very-low-dose twice-daily aspirin maintains platelet inhibition and improves haemostasis during dual antiplatelet therapy for acute coronary syndrome. Platelets 30, 148-157. doi: 10.1080/09537104.2019.1572880

Pascale, S., Petrucci, G., Dragani, A., Habib, A., Zaccardi, F., Pocaterra, D., et al. (2012). Aspirin-insensitive thromboxane biosynthesis in essential thrombocythemia is explained by accelerated renewal of the drug target. Blood 119, 3595-3603. doi: 10.1182/blood-2011-06-359224

Patrignani, P., Filabozzi, P., and Patrono, C. (1982). Selective cumulative inhibition of platelet thromboxane production by low-dose aspirin in healthy subjects. $J$. Clin. Invest. 69, 1366-1372. doi: 10.1172/JCI110576

Patrignani, P., Morton, H., Cirino, M., Lord, A., Charette, L., Gillard, J., et al. (1989). Fractional conversion of thromboxane A2 and B2 to urinary 2,3-dinorthromboxane B2 and 11-dehydro-thromboxane B2 in the cynomolgus monkey. Biochim. Biophys. Acta 992, 71-77. doi: 10.1016/0304-4165(89)90052-4

Patrono, C. (1994). Aspirin as an antiplatelet drug. N. Engl. J. Med. 330, 1287-1294. doi: 10.1056/NEJM199405053301808

Patrono, C., and Baigent, C. (2014). Nonsteroidal anti-inflammatory drugs and the heart. Circulation 129, 907-916. doi: 10.1161/CIRCULATIONAHA.113.004480

Patrono, C., Ciabattoni, G., Pinca, E., Pugliese, F., Castrucci, G., De Salvo, A., et al. (1980). Low-dose aspirin and inhibition of thromboxane B2 production in healthy subjects. Thromb. Res. 17,317-327. doi: 10.1016/0049-3848(80)90066-3

Patrono, C., Ciabattoni, G., Pugliese, F., Pierucci, A., Blair, I. A., and FitzGerald, G. A. (1986). Estimated rate of thromboxane secretion into the circulation of normal man. J. Clin. Invest. 77, 590-594. doi: 10.1172/JCI112341

Patrono, C., Ciabattoni, G., Remuzzi, G., Gotti, E., Bombardieri, S., Di Munno, O., et al. (1985). Functional significance of renal prostacyclin and thromboxane A2 production in patients with systemic lupus erythematosus. J. Clin. Invest. 76, 1011-1018. doi: 10.1172/JCI112053

Patrono, C., García Rodríguez, L. A., Landolfi, R., and Baigent, C. (2005). Lowdose aspirin for the prevention of atherothrombosis. N. Engl. J. Med. 353, 2373-2383. doi: 10.1056/NEJMra052717

Patrono, C., Baigent, C., Hirsh, J., and Roth, G. (2008). Antiplatelet drugs: American College of Chest Physicians Evidence-Based Clinical Practice Guidelines (8th Edition). Chest 133, 199S-233S. doi: 10.1378/chest.08-0672

Patrono, C., and Rocca, B. (2008). Aspirin: promise and resistance in the new millennium. Arterioscler. Thromb. Vasc. Biol. 28, 25S-32S. doi: 10.1161/ ATVBAHA.107.160481

Patrono, C., and Rocca, B. (2017). Type 2 diabetes, obesity, and aspirin responsiveness. J. Am. Coll. Cardiol. 69, 613-615. doi: 10.1016/j.jacc.2016.11.049

Patrono, C., Rocca, B., and De Stefano, V. (2013). Platelet activation and inhibition in polycythemia vera and essential thrombocythemia. Blood 121, 1701-1711. doi: 10.1182/blood-2012-10-429134

Pedersen, A. K., and FitzGerald, G. A. (1984). Dose-related kinetics of aspirin. Presystemic acetylation of platelet cyclooxygenase. N. Engl. J. Med. 311, 12061211. doi: 10.1056/NEJM198411083111902

Petrucci, G., Rizzi, A., Cavalca, V., Habib, A., Pitocco, D., Veglia, F., et al. (2016). Patient-independent variables affecting the assessment of aspirin responsiveness by serum thromboxane measurement. Thromb. Haemost. 116, 891-896. doi: 10.1160/TH16-05-0349

Petrucci, G., Zaccardi, F., Giaretta, A., Cavalca, V., Capristo, E., Cardillo, C., et al. (2019). Obesity is associated with in vivo platelet activation and impaired responsiveness to once-daily, low-dose aspirin. J. Thromb. Haemost. 17, 885895. doi: $10.1111 /$ jth. 14445

Pierucci, A., Simonetti, B. M., Pecci, G., Mavrikakis, G., Feriozzi, S., Cinotti, G. A., et al. (1989). Improvement of renal function with selective thromboxane antagonism in lupus nephritis. N. Engl. J. Med. 320, 421-425. doi: 10.1056/ NEJM198902163200703

Reilly, I. A., and FitzGerald, G. A. (1987). Inhibition of thromboxane formation in vivo and ex vivo: implications for therapy with platelet inhibitory drugs. Blood 69, 180-186.

Remuzzi, G., FitzGerald, G. A., and Patrono, C. (1992). Thromboxane synthesis and action within the kidney. Kidney Int. 41, 1483-1493. doi: 10.1038/ ki.1992.217

Reny, J. L., Berdagué, P., Poncet, A., Barazer, I., Nolli, S., Fabbro-Peray, P., et al. (2012). Antiplatelet drug response status does not predict recurrent ischemic 
events in stable cardiovascular patients: results of the Antiplatelet Drug Resistances and Ischemic Events study. Circulation 125, 3201-3210. doi: 10.1161/CIRCULATIONAHA.111.085464

Roberts, L. J., 2nd, Sweetman, B. J., and Oates, J. A. (1981). Metabolism of thromboxane B2 in man. Identification of twenty urinary metabolites. J. Biol. Chem. 256, 8384-8393.

Rocca, B., Ciabattoni, G., Tartaglione, R., Cortellazzo, S., Barbui, T., Patrono, C., et al. (1995). Increased thromboxane biosynthesis in essential thrombocythemia. Thromb. Haemost. 74, 1225-1230. doi: 10.1055/s-0038-1649916

Rocca, B., and Patrono, C. (2005). Determinants of the interindividual variability in response to antiplatelet drugs. J. Thromb. Haemost. 3, 1597-1602. doi: $10.1111 /$ j.1538-7836.2005.01380.x

Rocca, B., and Patrono, C. (2015). Platelet progenitors: the hidden drug target. Eur. Heart J. 36, 3211-3213. doi: 10.1093/eurheartj/ehv366

Rocca, B., Santilli, F., Pitocco, D., Mucci, L., Petrucci, G., Vitacolonna, E., et al. (2012). The recovery of platelet cyclooxygenase activity explains interindividual variability in responsiveness to low-dose aspirin in patients with and without diabetes. J. Thromb. Haemost. 10, 1220-1230. doi: 10.1111/j.1538-7836.2012.04723.x

Rocca, B., Fox, K. A. A., Ajjan, R. A., Andreotti, F., Baigent, C., Collet, J.-P., et al. (2018). Antithrombotic therapy and body mass: an expert position paper of the ESC Working Group on Thrombosis. Eur. Heart J. 39, 1672-1686f. doi: 10.1093/eurheartj/ehy066

Rothwell, P. M., Algra, A., Chen, Z., Diener, H. C., Norrving, B., and Mehta, Z. (2016). Effects of aspirin on risk and severity of early recurrent stroke after transient ischaemic attack and ischaemic stroke: time-course analysis of randomised trials. Lancet 388, 365-375. doi: 10.1016/S0140-6736(16)30468-8

Rothwell, P. M., Cook, N. R., Gaziano, J. M., Price, J. F., Belch, J. F. F., Roncaglioni, M. C., et al. (2018). Effects of aspirin on risks of vascular events and cancer according to bodyweight and dose: analysis of individual patient data from randomised trials. Lancet 392, 387-399. doi: 10.1016/S0140-6736(18)31133-4

Santilli, F., Rocca, B., De Cristofaro, R., Lattanzio, S., Pietrangelo, L., Habib, A., et al. (2009). Platelet cyclooxygenase inhibition by low-dose aspirin is not reflected consistently by platelet function assays. Implications for aspirin "resistance". J. Am. Coll. Cardiol. 53, 667-677. doi: 10.1016/j.jacc.2008.10.047

Smith, J. B., and Willis, A. L. (1971). Aspirin selectively inhibits prostaglandin production in human platelets. Nat. New Biol. 231, 235-237. doi: 10.1038/ newbio231235a 0
Smith, J. P., Haddad, E. V., Taylor, M. B., Oram, D., Blakemore, D., Chen, Q., et al. (2012). Suboptimal inhibition of platelet cyclooxygenase-1 by aspirin in metabolic syndrome. Hypertension 59, 719-725. doi: 10.1161/ HYPERTENSIONAHA.111.181404

Spectre, G., Arnetz, L., Ostenson, C. G., Brismar, K., Li, N., and Hjemdahl, P. (2011). Twice daily dosing of aspirin improves platelet inhibition in whole blood in patients with type 2 diabetes mellitus and micro- or macrovascular complications. Thromb. Haemost. 106, 491-499. doi: 10.1160/TH11-04-0216

Tefferi, A., Vannucchi, A. M., and Barbui, T. (2018). Polycythemia vera treatment algorithm 2018. Blood Cancer J. 8, 3. doi: 10.1038/s41408-017-0042-7

The RISC Group. (1990). Risk of myocardial infarction and death during treatment with low dose aspirin and intravenous heparin in men with unstable coronary artery disease. Lancet 336, 827-830. doi: 10.1016/0140-6736(90)92336-G

van Kooten, F., Ciabattoni, G., Patrono, C., Dippel, D. W. J., and Koudstaal, P. J. (1997). Platelet activation and lipid peroxidation in patients with acute ischemic stroke. Stroke 28, 1557-1563. doi: 10.1161/01.STR.28.8.1557

Vejar, M., Fragasso, G., Hackett, D., Lipkin, P. D., Maseri, A., Born, G. V., et al. (1990). Dissociation of platelet activation and spontaneous myocardial ischemia in unstable angina. Thromb. Haemost. 63, 163-168. doi: 10.1055/ s-0038-1645038

Working Group on Aspirin Resistance (2005). Aspirin resistance: position paper of the Working Group on Aspirin Resistance. J. Thromb Haemost. 3, 1309-1311.

Conflict of Interest: $\mathrm{CP}$ reports consulting and lecture fees from Acticor Biotech, Amgen, Bayer, GlaxoSmithKline and Zambon, and institutional research grants from Bayer, Cancer Research UK (Catalyst Award-Aspirin for Cancer Prevention Collaboration), European Commission, and Italian Drug Agency (AIFA); he serves as the chairperson of the Scientific Advisory Board of the International Aspirin Foundation. BR reports consulting and lecture fees from Bayer, institutional research grants from Cancer Research UK (Catalyst Award-Aspirin for Cancer Prevention Collaboration), and from the Italian Drug Agency (AIFA).

Copyright $\odot 2019$ Patrono and Rocca. This is an open-access article distributed under the terms of the Creative Commons Attribution License (CC BY). The use, distribution or reproduction in other forums is permitted, provided the original author(s) and the copyright owner(s) are credited and that the original publication in this journal is cited, in accordance with accepted academic practice. No use, distribution or reproduction is permitted which does not comply with these terms. 